Bibliographic Review

\section{Resumo}

Os estudos sobre composição isotópica de nitrogênio em solo e planta são importantes para identificar diferenças nos processos de ciclagem biogeoquímica deste elemento em ecossistemas. Para avaliar a contribuição da FBN, bem como, as

\title{
Estudo do fracionamento isotópico de nitrogênio aplicado à gramíneas e leguminosas forrageiras
}

Édina Cristine Pereira Lopes ${ }^{1}$ Anibal de Moraes $^{2}$ Claudete Reisdorfer Lang ${ }^{2}$ diferenças na capacidade de fixar e transferir $\mathrm{N}$ entre espécies de leguminosas perenes e gramíneas existem várias técnicas que tem sido utilizadas, como a redução de acetileno, diluição isotópica de ${ }^{15} \mathrm{~N}$ e abundância natural de ${ }^{15} \mathrm{~N}$. No entanto, as características responsáveis por estas variações precisam ser, em grande parte, identificadas. Esta revisão teve como objetivo comparar a dinâmica de transferência e fixação de $\mathrm{N}$ entre gramíneas e leguminosas forrageiras.

Palavras chave: técnicas isotópicas, abundância natural de ${ }^{15} \mathrm{~N}, \mathrm{FBN}$

\section{Study of isotopic fractionation of nitrogen applied to grasses and forage legumes}

\begin{abstract}
Studies about isotopic composition of nitrogen in soil and plant matter to identify differences in biogeochemical cycling processes of this element in ecosystems. To assess the contribution of BNF, as well as differences in ability to fix and transfer $\mathrm{N}$ among species of perennial leguminous crops and grasses there are several techniques that have been used as the acetylene reduction, isotopic dilution ${ }^{15} \mathrm{~N}$ and ${ }^{15} \mathrm{~N}$ natural abundance. However, the features responsible for these changes need to be largely identified. This review aimed to compare the dynamics of transfer and fixing $\mathrm{N}$ between grasses and forage legumes.
\end{abstract}

Key words: isotopic techniques, ${ }^{15} \mathrm{~N}$ natural abundance, BNF

\section{Estudio del fraccionamiento isotópico del nitrógeno aplicado a las gramíneas y leguminosas forrajeras}

\section{Resumen}

Los estudios de la composición isotópica del nitrógeno en el suelo y las plantas son importantes para identificar las diferencias en los procesos biogeoquímicos de reciclage de este elemento en los ecosistemas. Para evaluar la contribución de BNF, así como las diferencias en la capacidad de fijar y transferir $\mathrm{N}$ entre las especies de legumbres perennes y gramíneas existen varias técnicas que se han utilizado, como la reducción de acetileno, dilución isotópica $15 \mathrm{~N}$ y abundancia natural de $15 \mathrm{~N}$. Sin embargo, las características responsables por estas variaciónes tienen que ser, en gran parte identificadas. Esta revisión tuvo como objetivo comparar la dinámica de la transferencia y la fijación de $\mathrm{N}$ de gramíneas y leguminosas forrajeras.

Palabras clave: técnicas isotópicas, abundancia natural de ${ }^{15} \mathrm{~N}, \mathrm{FBN}$

\footnotetext{
Received at: 11/11/15 Accepted for publication at: 27/03/16

1 Eng. Dr. Doutorando em Agronomia, Universidade Federal do Paraná -UFPR, Curitiba-Pr. Email: edinacplopes@gmail.com.

2 Prof Dr. Departamento de Fitotecnia e Fitossanitarismo, Universidade Federal do Paraná UFPR, Curitiba-Pr. Email:anibaldemoraes@ gmail.com; claudetelangr@gmail.com
} 


\section{Introdução}

$\mathrm{O}$ nitrogênio $(\mathrm{N})$ é o componente mais abundante na atmosfera, encontrando-se numa forma combinada $\left(\mathrm{N}_{2}\right)$ que as plantas não são capazes de utilizar. Nos sistemas terrestres, é o elemento químico mais limitante para o crescimento vegetal (VITOUSEK e FARRINGTON, 1997). As plantas requerem nitrogênio em quantidade superior a qualquer outro nutriente mineral, e a disponibilidade deste nutriente geralmemte limita a produtividade das plantas em muitos ecossistemas naturais e agrícolas (EPSTEIM e BLOOM, 2005).

$\mathrm{O} N$ é um dos principais bioelementos, constituindo os aminoácidos e ácidos nucleicos e outras moléculas de plantas. Em particular, as leguminosas forrageiras têm um elevado teor de proteínas (14-29 \% em alfafa) (MAURIES, 2003). As leguminosas foram usadas em rotações ou associadas a gramíneas na agricultura tradicional, desde os tempos antigos, por causa de sua capacidade de fixar o nitrogênio atmosférico. No entanto, a disponibilidade de $\mathrm{N}$ sintético relativamente barato no mundo industrializado durante o século XX levou a uma diminuição da sua utilização. Na verdade, o uso excessivo de fertilizantes resultaram em uma "cascata de N", ou seja, vazamentos de nitrogênio para o meio ambiente, causando diversos efeitos adversos sobre os ecossistemas e a saúde humana (SUTTON e VAN GRINSVEN, 2011).

Atualmente, a reintrodução de leguminosas e o uso da Fixação Biológica de Nitrogênio (FBN) como uma fonte de $\mathrm{N}$ parece ser uma ferramenta que pode ajudar a reduzir o custo de produção e também a preservar o ambiente. A disponibilidade de $\mathrm{N}$ é depois de deficiência hídrica e as condições climáticas, o principal fator limitante do crescimento das plantas. O fornecimento suficiente de $\mathrm{N}$ é de suma importância para o metabolismo da planta, e a adição de $\mathrm{N}$ aumenta a eficiência da fotossíntese (SUTTON e VAN GRINSVEN, 2011). Em sistemas agrícolas, em parte do sistema solo-planta, o N é acumulado na safra e removidos na colheita. Por conseguinte, quantidades consideráveis de $\mathrm{N}$ necessitam serem fornecidas para atingir o rendimento máximo (LEA e MOROT-GAUDRY, 2001).

As principais fontes de $\mathrm{N}$ reativo na agricultura são fertilizantes sintéticos, FBN e adubo orgânico (estrume animal). Em termos quantitativos, fertilizantes sintéticos produzidos por meio do processo de Haber-Bosch é a forma mais consumida de N. No ano 2000, cerca de 100 megatonelas de fertilizantes industriais foram utilizados no mundo. Na verdade, o uso de fertilizantes sintéticos é grande responsável pelo aumento da produção agrícola mundial, que ocorreu durante o século 20. Estercos de gado são também uma importante fonte de $\mathrm{N}$ para a agricultura. Estima-se que 140 milhões de toneladas de $\mathrm{N}$ foram produzidos no mundo no ano 2000 (SUTTON e VAN GRINSVEN, 2011).

A fixação biológica de nitrogênio é a terceira fonte de $\mathrm{N}$ mais utilizada na agricultura. A FBN foi recentemente estimada em torno de 40-60 megatonelas de N por ano no mundo (SUTTON e VAN GRINSVEN, 2011). A fixação biológica de nitrogênio é um processo natural de redução de diazoto molecular $\left(\mathrm{N}_{2}\right)$ em amonia $\left(\mathrm{NH}^{3}\right)$, realizada por uma série de espécies de bactérias chamadas diazotrofos, graças à sua enzima nitrogenase, que catalisa a reação:

$$
\mathrm{N}_{2}+3 \mathrm{H}_{2} \rightarrow 2 \mathrm{NH}_{3}
$$

Assim, o $\mathrm{N}_{2}$, é convertido para uma forma que pode ser utilizada por sistemas biológicos (LEA e MOROT-GAUDRY, 2001). Os organismos fixadores de N2 abrangem formas de vida livre, associações simbióticas com plantas, autótrofos e heterótrofos. Diazotrofos simbióticos incluem uma série de gêneros da Rhizobiaceae (por exemplo Sinorhizobium, Mesorhizobium, Bradyrhizobium), que formam simbioses com as leguminosas. O Rhizobium provoca sobre as raízes do hospedeiro (leguminosa) a formação de nódulos, que são órgãos especializados os quais contêm as bactérias fixadoras de $\mathrm{N}_{2}$ Essa interação simbiótica é altamente específica. Um determinado rizóbio só forma nódulos em um número restrito de espécies. A fixação simbiótica de $\mathrm{N}_{2}$ em leguminosas é responsável por grandes contribuições da fixação de $\mathrm{N}$ em sistemas de cultivo. Particularmente, as leguminosas forrageiras são responsáveis por uma fixação em um intervalo de $50-250 \mathrm{~kg} \mathrm{~N}^{-1}$ por ano (UNKOVICH et al., 2008), com um valor médio de $180 \mathrm{~kg} \mathrm{~N} \mathrm{ha}^{-1}$ para alfafa (LOUARN et al., 2010). A contribuição da FBN nas plantas não-leguminosas é comparativamente menor do que a contribuição nas plantas leguminosas.

Desde os primeiros trabalhos deDOBEREINER (1961) isolando bactérias fixadoras de N2 a partir das raízes de cana-de-açúcar, algumas bactérias diazotróficas têm sido associadas com gramíneas em geral. URQUIAGA et al (2011), avaliando 6 variedades comerciais de cana-de-açúcar, observaram que as mesmas foram capazes de obter pela FBN de 40,2 a $64,0 \mathrm{~kg} \mathrm{ha}^{-1}$ de $\mathrm{N}$. 
Estudo do fracionamento isotópido de...

Study of isotopic fractionation of...

Estudio del fraccionamiento isotópico del...
Estudos com outros tipos de gramíneas têm sido realizados, como no caso do sorgo e milheto (LEE et al., 1994) e arroz (WATANABE et al., 1987). MORAIS et al. (2009) avaliaram os genótipos de capim-elefante (Cameroon, BAG 02, Gramafante, Roxo e CNPGL F06-3) em solos de baixa fertilidade, e verificaram que a FBN foi responsável em média por $51 \%$ do $\mathrm{N}$ assimilado pela planta. Em outro estudo, MORAIS et al. (2011) conduziram três experimentos a campo em solos de baixa fertilidade, dois no Rio de Janeiro e um no Espírito Santo, com as variedades Gramafante, Cameroon, BAG 02, CNPGL F06-3, CNPGL F79-2 e Roxo, e quatro dos genótipos avaliados obtiveram de 18 a $70 \%$ do $\mathrm{N}$ derivado da FBN.

Para avaliar a contribuição da FBN, bem como, as diferenças na capacidade de fixar e transferir N entre espécies de leguminosas perenes e gramíneas existem várias técnicas que tem sido utilizadas, como a redução de acetileno, diluição isotópica de ${ }^{15} \mathrm{~N}$ e abundância natural de ${ }^{15} \mathrm{~N}$. No entanto, as características responsáveis por estas variações precisam ser, em grande parte, identificadas. Esta revisão teve como objetivo comparar a dinâmica de transferência e fixação de $\mathrm{N}$ entre gramíneas e leguminosas forrageiras.

\section{Desenvolvimento}

Os estudos sobre composição isotópica de nitrogênio em solo e planta são importantes para identificar diferenças nos processos de ciclagem biogeoquímica deste elemento em ecossistemas. De acordo com CERETTA e GIROTTO (2011) uma das grandes dúvidas em relação à adubação nitrogenada é quanto realmente as plantas absorvem de fontes minerais ou orgânicas de N. Esse problema pode ser solucionado com o uso da técnica isotópica de $\mathrm{N}$ marcado, que utiliza isótopos estáveis de N (15N) como traçador, cujo fluxo de passagem e os sítios de residência podem ser identificados.

Os isótopos são formas do mesmo elemento químico que diferem no número de neutrons. À medida que pertencem ao mesmo elemento, que tem o mesmo número de protons (número atômico $\mathrm{Z})$. O número de neutrons $(\mathrm{N})$ afeta o número total de núcleos que determina o número de massa $(\mathrm{A}=\mathrm{Z}+\mathrm{N})$. O número de massa identifica cada isótopo. Alguns isótopos são radioativos, eles têm um núcleo instável que se desintegra emitindo radiação espontaneamente, enquanto outros, chamados de isótopos estáveis, não são objeto de decadência e não são radioativos.

Os isótopos nitrogênio-14, nitrogênio-15 e nitrogênio-16 são três isótopos do elemento nitrogênio com massa atômica 14,15 e 16 , respectivamente. ${ }^{14} \mathrm{~N}$ $\mathrm{e}^{15} \mathrm{~N}$ são isótopos estáveis de nitrogênio, enquanto o resto são radiaotivos. $\mathrm{O}^{14} \mathrm{~N}$ mais leve, é naturalmente, muito mais abundante do que ${ }^{15} \mathrm{~N}$ constituindo 99,63\% do nitrogênio natural (Figura 1).

Entradas de $\mathrm{N}$ são necessárias para substituir o $\mathrm{N}$ perdido ou removido, a fim de manter a produtividade em sistemas agrícolas. Os principais métodos de quantificação podem ser classificados em métodos diretos ou indiretos sobre as abordagens de medição de fixação de $\mathrm{N}_{2}$.

Os métodos diretos (incorporação de ${ }^{15} \mathrm{~N}_{2^{\prime}}$ ensaio de redução de acetileno e método evolução de hidrogênio) fornecem uma medida direta da atividade da nitrogenase e, portanto, as taxas instantâneas de fixações de $\mathrm{N}_{2}$ absolutas. No entanto, elas não podem ser usadas no campo para obter a quantidade de $\mathrm{N}_{2}$ fixada ao longo de um ano. A maioria dos métodos indiretos se baseiam na separação de $\mathrm{N}$ na planta para a fração feita a partir do solo e a fração derivada da atmosfera e requerem a utilização de plantas não fixadoras de $\mathrm{N}$ como controle. Isto implica na limitação de seleção de plantas com uma taxa de absorção de N semelhante a de leguminosas, que é dada principalmente pelo seu enraizamento, morfologia e profundidade.

A diferença de $\mathrm{N}$-total do sistema solo-planta é um método indireto que se baseia na medição da diferença de $\mathrm{N}$ total entre plantas fixadoras de $\mathrm{N}$ e plantas não-fixadoras de N. Isto porque as diferenças entre teores de $\mathrm{N}$ são geralmente pequenas e precisam serem medidas ao longo de vários anos para uma quantificação precisa. Finalmente, técnicas isotópicas de ${ }^{15} \mathrm{~N}$ baseiam-se no fato de que as diferentes fontes de $\mathrm{N}$ para uma leguminosa (do solo e do ar) exibem diferentes abundâncias isotópicas. Isso torna possível calcular a fixação de $\mathrm{N}_{2}$, comparando a composição de ${ }^{15} \mathrm{~N}$ de uma planta fixadora de $\mathrm{N}_{2^{\prime}}$ com uma planta não- fixadora de $\mathrm{N}_{2}$ (que só usa o $\mathrm{N}$ do solo) e uma planta fixadora de $\mathrm{N}$ mas sem fornecimento de $\mathrm{N}$ no solo (SHEARER e KOHL, 1986; UNKOVICH et al, 2008) (Figura 2). 


\begin{tabular}{|c|c|c|c|}
\hline \multicolumn{2}{|c|}{ Stable } & Radioactive & \\
\hline & & & $\begin{array}{ll}\text { - } & \text { Protons } \\
\text { - Neutons } \\
\text { - Electrons }\end{array}$ \\
\hline${ }^{14} \mathrm{~N}$ & ${ }^{15} \mathrm{~N}$ & ${ }^{16} \mathrm{~N},{ }^{17} \mathrm{~N} \ldots{ }^{25} \mathrm{~N}$ & \\
\hline $\begin{array}{l}Z=7 \\
N=7 \\
A=14\end{array}$ & $\begin{array}{l}Z=7 \\
N=8 \\
A=15\end{array}$ & $\begin{array}{l}Z=7 \\
N=9 \\
A=16\end{array}$ & $\begin{array}{l}Z=\text { protons } \\
N=\text { neutrons } \\
A=\text { atomic mass }\end{array}$ \\
\hline $99.63 \%$ & $0.37 \%$ & & Atom $\%{ }^{15} \mathrm{~N}$ \\
\hline
\end{tabular}

Figura 1. Os isótopos de nitrogênio.

Técnicas isotópicas de ${ }^{15} \mathrm{~N}$ e método abundância natural $d e{ }^{15} \mathrm{~N}$.

As técnicas isotópicas de ${ }^{15} \mathrm{~N}$ são muito úteis para quantificar a contribuição da FBN associadas as não-leguminosas, e englobam a contribuição da FBN no ciclo completo das culturas. Especificamente, a técnica baseada na abundância natural de ${ }^{15} \mathrm{~N}$ é mais apropriada para avaliações em sistemas agrícolas e, quando aplicadas adequadamente, os resultados são mais precisos (BALDANI et al., 2009).

A ocorrência natural do isótopo menor $\left({ }^{15} \mathrm{~N}\right)$ em relação ao total de isótopos de longa duração do elemento $\left({ }^{15} \mathrm{~N}\right)$ dada em unidades de Atom $\%{ }^{15} \mathrm{~N}$ ou $\delta{ }^{15} \mathrm{~N}$ é chamada de abundância isotópica de ${ }^{15} \mathrm{~N}$ ou abundância natural de ${ }^{15} \mathrm{~N}$.

As composições de isótopos estáveis de materiais naturais são usados como traçadores para a compreensão dos pontos de origem de um elemento em um processo ou em um ciclo no ambiente (FRY, 2006). Quanto aos estudos de fixação de $\mathrm{N}_{2}$, os isótopos de $\mathrm{N}$ servem para identificar a fonte de $\mathrm{N}$ na planta: o $\mathrm{N}_{2}$ fixa a partir da atmosfera ou $\mathrm{N}$ é assimilado a partir do solo.

Técnicas isotópicas de quantificação da fixação de $\mathrm{N}_{2}\left({ }^{15} \mathrm{~N}\right)$, baseiam-se no princípio de que, se as concentrações de ${ }^{15} \mathrm{~N}$ da planta, o $\mathrm{N}$ disponível do solo e $\mathrm{N}_{2}$ atmosférico são conhecidos a composição ${ }^{15} \mathrm{~N}$ da planta é medida, sendo assim, é possível calcular a percentagem de $\mathrm{N}$ derivado da atmosfera (\% Ndfa) da planta N (Figura 4). De um modo geral, o $\mathrm{N}$ mineral do solo é ligeiramente enriquecido em ${ }^{15} \mathrm{~N}$ em comparação com o ambiente, no entanto esta diferença é muito pequena.

$\mathrm{O}$ método de diluição isotópica de ${ }^{15} \mathrm{~N}$ consiste em enriquecer o solo artificialmente pela adição de fertilizantes marcados com ${ }^{15} \mathrm{~N}$ para fazer esta diferença maior. O método da abundância natural de ${ }^{15} \mathrm{~N}$ usa diretamente a diferença natural que ocorre entre o solo e as concentrações de ${ }^{15} \mathrm{~N}$ atmosfera para distinguir a origem do $\mathrm{N}$ da leguminosa (BERGENSEN e TURNER, 1983; SHEARER e KOHL, 1986).

A principal limitação do método abundância natural ${ }^{15} \mathrm{~N}$ é que a diferença de abundância de ${ }^{15} \mathrm{~N}$ de plantas fixadoras de $\mathrm{N}_{2}$ e plantas de referência não fixadoras de $\mathrm{N}_{2}$ é muito pequena, normalmente inferior a $10 \%$. Essa diferença tem um impacto direto sobre as estimativas Ndfa \% (Figura 4): quanto maior a diferença, maior será a precisão do Ndfa \%. Se a diferença é $<2 \%$, o método ${ }^{15} \mathrm{~N}$ não pode ser utilizado. Em particular, a variabilidade de ${ }^{15} \mathrm{~N}$ de dois parâmetros deve ser menor do que a diferença entre eles. 
Estudo do fracionamento isotópido de...

Study of isotopic fractionation of..

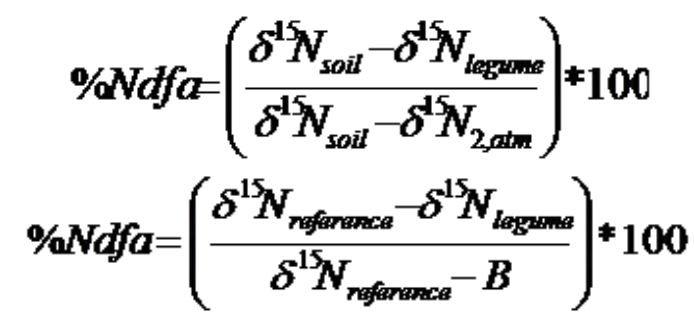

$\delta 15 \mathrm{~N}$ soil- $-\delta^{15} \mathrm{~N}$ reference non-fixing plant

$\delta 15 \mathrm{~N}_{2}$, atm $-\mathrm{B}=\delta^{15} \mathrm{~N}$ shoots of a legume growing without $\mathrm{N}$.

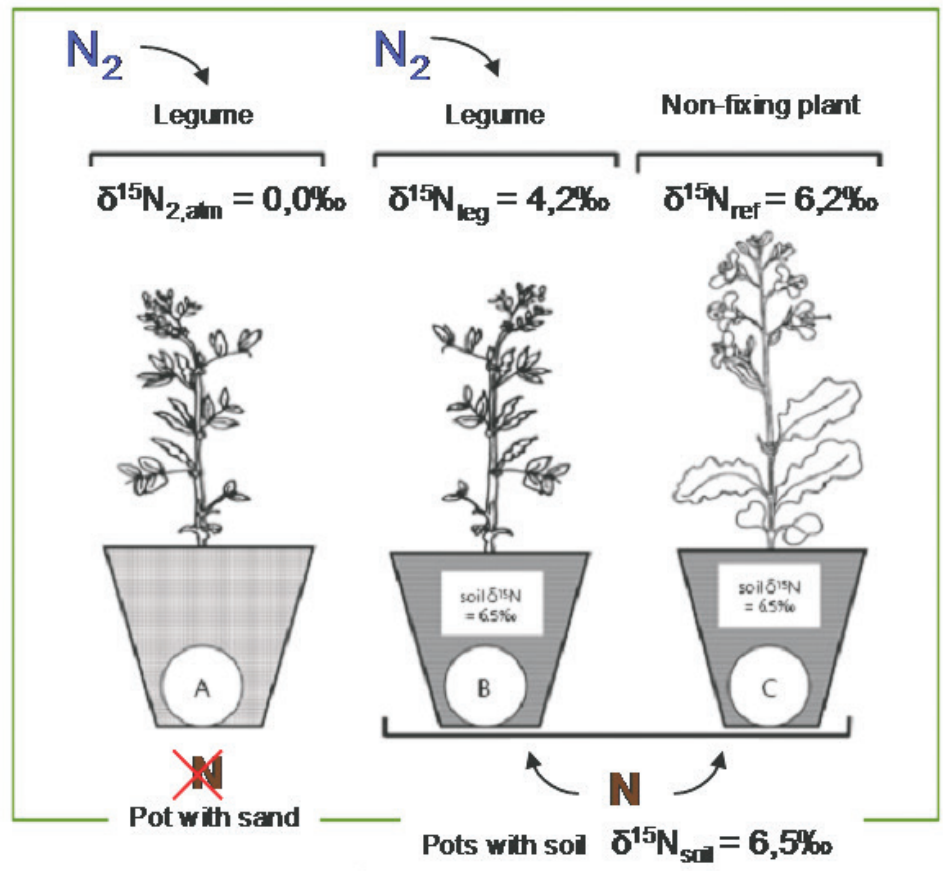

Figura 2. Cálculo de Ndfa \% (porcentagem de plantas com N derivado N2 atmosférico). Conforme a Figura 2, três indivíduos são necessários para o cálculo de Ndfa \%. Uma associação entre leguminosa e Rhizobium que utiliza uma combinação de $\mathrm{N}$ do solo e $\mathrm{N}_{2}$ atmosférico terá uma composição ${ }^{15} \mathrm{~N}$ situada entre o $\mathrm{N}$ do solo e o N do ar. A contribuição proporcional da FBN para fixação do $\mathrm{N}$ em leguminosa pode ser calculada a partir da abundância de ${ }^{15} \mathrm{~N}$ da leguminosa, isso se as abundâncias de ${ }^{15} \mathrm{~N}$ de solo disponíveis para as plantas e $\mathrm{N}^{2}$ atmosférico sejam conhecidos. Na prática, a composição de ${ }^{15} \mathrm{~N}$ de plantas de referência é utilizada em vez da abundância de ${ }^{15} \mathrm{~N}$ do pool de $\mathrm{N}$ no solo. Da mesma forma, $\delta{ }^{15} \mathrm{~N}$ de $\mathrm{N}_{2}$ atmosférico é substituído pelo $\delta{ }^{15} \mathrm{~N}$ de uma leguminosa nodulada crescendo em um meio livre de $\mathrm{N}$ mineral, tão completamente dependentes de fixação de $\mathrm{N}_{2}$. Plantas de referência refletem bem a composição isotópica do solo, como eles parecem não discriminar entre ${ }^{14} \mathrm{~N}$ e ${ }^{15} \mathrm{~N}$ durante a assimilação do $\mathrm{N}$ mineral. O uso de plantas de referência é conveniente, por várias razões: (1) a composição do $\delta{ }^{15} \mathrm{~N}$ do $\mathrm{N}$ total do solo é dominado por formas estáveis de $\mathrm{N}$ e não é um guia confiável para $\delta{ }^{15} \mathrm{~N}$ da planta com $\mathrm{N}$ mineral disponível (principalmente $\mathrm{NO}^{3-}$ e $\mathrm{NH}^{4+}$ ), (2) a abundância do solo é tecnicamente mais difícil de medir (3) representam as variações temporais $\delta^{15} \mathrm{~N}$, bem como fracionamento isotópicos dentro transformações do solo. Por outro lado, plantas de referência devem ser totalmente dependentes de $\mathrm{N}$ mineral do solo, usando os mesmos pools de $\mathrm{N}$ do solo nas concentrações de ${ }^{15} \mathrm{~N}$ idênticos (crescendo no mesmo local) e ter profundidade de enraizamento semelhante, padrão de absorção de $\mathrm{N}$ e crescimento como a planta fixadora de $\mathrm{N}_{2}$.

Applied Research \& Agrotechnology v9 n1 jan/apr. (2016)

Print-ISSN 1983-6325 (On line) e-ISSN 1984-7548 
Fracionamento isotópico de ${ }^{15} \mathrm{~N}$

O fracionamento isotópico é um fenômeno onde as concentrações isotópicas dos reagentes e produtos difere progressivamente como uma transformação ocorre devido às ligeiramente diferentes propriedades físicas dos isótopos. Devido às suas grandes massas, isótopos mais pesados tendem a ter taxas mais baixas de difusão e baixas constantes de equilíbrio para uma reação. Os isótopos podem apresentar um comportamento químico quase idêntico sobre as propriedades químicas, uma vez que estes são determinados pela estrutura eletrônica, e os isótopos podem compartilhar o número de prótons e elétrons. O fator de fracionamento isotópico $\beta$ expressa a magnitude do efeito isotópico cinético.
Discriminação isotópica entre ${ }^{14} \mathrm{~N}$ e ${ }^{15} \mathrm{~N}$ está envolvida em transformações dentro do ciclo N. Estes problemas conduzem a pequenas variações $\delta$ $15 \mathrm{~N}$, cuja magnitude é importante no contexto do método $\delta{ }^{15} \mathrm{~N}$. Particularmente, a variabilidade da abundância de $15 \mathrm{~N}$ dos brotos da leguminosa (valor B) tem impacto sobre a precisão nas estimativas de \% Ndfa. O valor B parece mudar em função de espécies de leguminosas e potencialmente com estirpes de rizóbios (STEELE et al., 1983.), Assim um novo valor " $\mathrm{B}$ " deve ser calculado para cada associação leguminosa-rizóbio. Além disso, dado o declínio progressivo da abundância de ${ }^{15} \mathrm{~N}$ na parte aérea (UNKOVICH et al., 1994) (Figura 3), as leguminosas devem ser colhidas no mesmo estágio de crescimento para calcular o valor " $\mathrm{B}$ ", que nada mais é que a
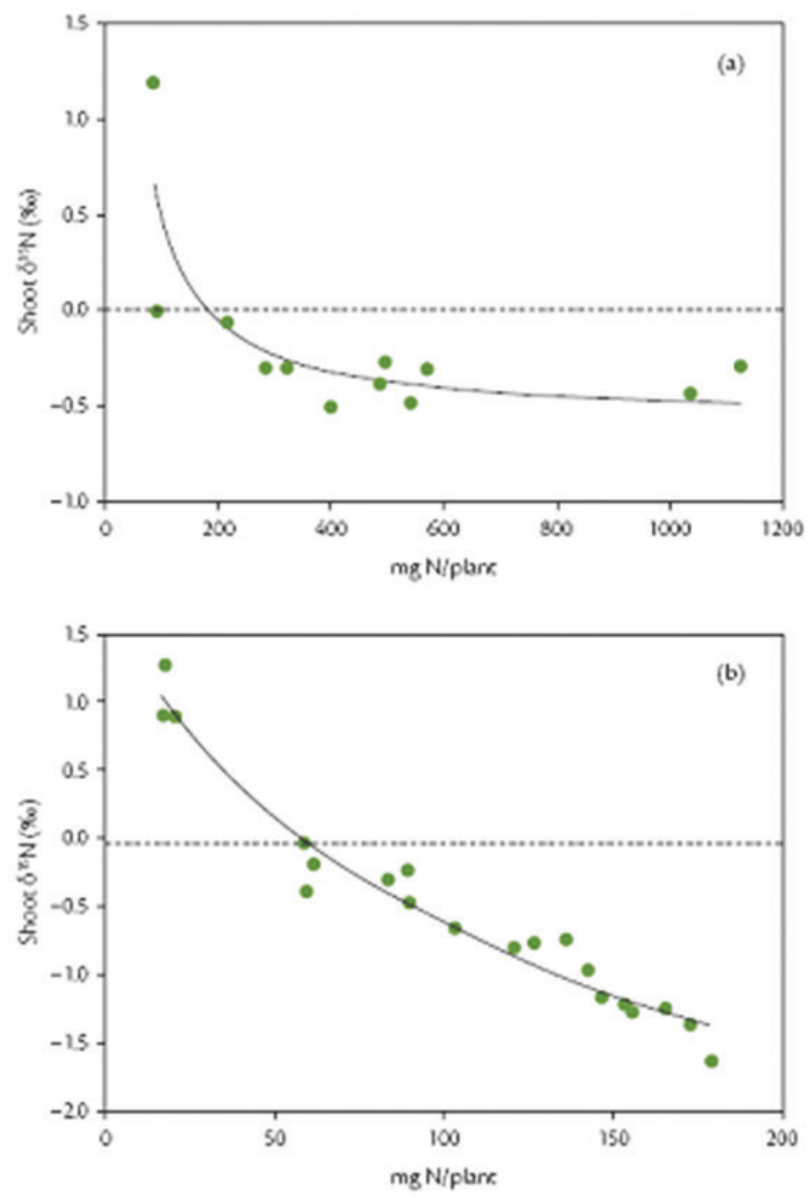

Figura 3. Mudanças dinâmicas na abundância de $\delta^{15} \mathrm{~N}$ de parte aérea de plantas sem fornecimento de $\mathrm{N}$ do solo (B) durante crescimento em (a) fava e (b) de grão de bico (UNKOVICH et. al., 1994.). 


\author{
Estudo do fracionamento isotópido de... \\ Study of isotopic fractionation of.. \\ Estudio del fraccionamiento isotópico del...
}

abundância isotópica de ${ }^{15} \mathrm{~N}$ dos órgãos acima do solo de uma leguminosa de crescimento completamente dependente de fixação de $\mathrm{N}_{2}$.

Além disso, quase todas as transformações do solo envolvem fracionamento isotópico em um ponto diferente. A nitrificação e desnitrificação são de grande importância com fatores isotópicos de fracionamento de $\beta=1,035$ e 1,02, respectivamente (MARIOTTI et al., 1982).

O primeiro passo do processo de nitrificação $\left(\mathrm{NH}_{4}+\right.$ a $\left.\mathrm{NO}_{2-}\right)$ leva o ${ }^{15} \mathrm{~N}$ do $\mathrm{NO}_{3-}$ empobrecido. Da mesma forma, as emissões de $\mathrm{N}_{2} \mathrm{O}$ e de $\mathrm{N}_{2}$ durante a desnitrificação são esgotadas em ${ }^{15} \mathrm{~N}$, o que resulta no enriquecimento de $\mathrm{NO}^{3-}$ no solo. Como ${ }^{15} \mathrm{~N}$ de derivados de $\mathrm{N}$ no solo é medida em planta não fixadora de $\mathrm{N}^{2}$, após o $\mathrm{N}$ foi assimilado, esses processos podem ser considerados como já realizados. Discriminações isotópicas também ocorrem in vivo durante a assimilação, bactérias fixadoras de $\mathrm{N}_{2}$ e metabolismo e transporte de N. A principal forma de $\mathrm{N}$ inorgânico assimilado pelas raízes das plantas é o nitrato $\left(\mathrm{NO}^{3-}\right)$ e amônio $\left(\mathrm{NH}^{4+}\right)$. O nitrato é transportado para o cloroplasto, onde é reduzida em nitrato redutase - redutase de nitrito de amoníaco. Amônio assimilado por bactérias simbióticas é transportado para dentro das células hospedeiras por transportadoras. Nos cloroplastos, de amoníaco a partir de diferentes origens é substrato da sintetase de glutamina e glutamina - 2 - oxoglutarato aminotransferase (GOGA) (EVANS, 2001).

Em plantas superiores, o fracionamento isotópico associado com redução de $\mathrm{NO}^{3-}$ geralmente não é expresso, porque todo o $\mathrm{NO}^{3-}$ tomado pela planta é assimilado e pode ser considerado como nula $(\beta=1,00025)$. $\mathrm{O} \mathrm{N}_{2}$ fixado mostra um pequeno efeito isótopo ( $\beta=1,009$ para Medicago sativa L.) (KYEIBOAHEN et al., 2002). Quanto as transformações metabólicas internas, as principais discriminações isotópicas têm sido observadas durante a reação GOGAT, que produz glutamato empobrecido em ${ }^{15} \mathrm{~N}$, deixando também glutaminas enriquecidas $\operatorname{com}{ }^{15} \mathrm{~N}$, a principal fonte primária de aminoácidos (WERNER e SCHMIDT, 2002). Discriminações também foram relatadas em reações de transaminação, tornando aminoácidos geralmente enriquecidos com ${ }^{15} \mathrm{~N}$ (HARE et al., 1991) e em biossíntese de clorofila, originário de plantas $\mathrm{C}^{3}$ esgotadas em ${ }^{15} \mathrm{~N}$ (CHIKARAISHI et al., 2005) .
Fracionamentos isotópicos in vivo podem traduzir em diferenças de $\delta^{15} \mathrm{~N}$ entre os órgãos da planta. Apesar da quantidade de $\delta^{15} \mathrm{~N}$ de plantas inteiras é normalmente próximo do $\delta 15 \mathrm{~N}$ do $\mathrm{N}_{2}$ atmosférico ( $0 \%$ o , o $\delta^{15} \mathrm{~N}$ dos nódulos é muitas vezes positiva (TJEPKEMA et al., 2000). Caules e raízes são os mais empobrecidos (SHEARER e KOHL, 1986) porque, na prática apenas a parte aérea é colhida e analisada, a $\delta^{15} \mathrm{~N}$ da parte aérea de leguminosas, que são totalmente dependentes de fixação do $\mathrm{N}_{2^{\prime}}$, chamado valor $\mathrm{B}$, este é usado em vez do $\delta^{15} \mathrm{~N}$ do ar em cálculos de \% Ndfa. Usando B garante que os tecidos amostrados de plantas fixadoras de $\mathrm{N}_{2}$ e plantas de referência desviar em $\delta^{15} \mathrm{~N}$ a partir da planta inteira com a mesma intensidade. Para não tomar em conta o fracionamento dentro do legume conduz a estimativas mais elevadas de Ndfa\% e o impacto torna-se importante quanto maior for a $\%$ Ndfa (BODDEY et al., 2000).

Os órgãos de uma planta suportam uma grande variação no teor de N. Estudar a composição isotópica dos órgãos $\left({ }^{15} \mathrm{~N}\right)$, é determinante para saber a sua concentração de $\mathrm{N}$, bem como a natureza das biomoléculas contendo $\mathrm{N}$ presentes neles. Uma vez que se conforma principalmente, proteínas estruturais e enzimáticas, um elevado teor de $\mathrm{N}$ é normalmente associado com os tecidos metabolicamente ativos, tais como meristemas e células fotossintéticos. Folhas ativas requerem quantidades consideráveis de proteínas, enzimas e pigmentos. Em contraste, os tecidos do caule estruturais têm pouco conteúdo de proteínas e, por conseguinte, uma baixa \% de N (LEA e MOROT-GAUDRY, 2001) .

Por outro lado, a \% N na planta também muda durante o crescimento, diminuindo com a colheita da biomassa (LEMAIRE e GASTAL, 1997). Isto acontece devido ao aumento de proporção tecidos do caule (diminuição da relação folha/colmo), bem como a uma diminuição em \% de $\mathrm{N}$ na fração de haste. Notavelmente, o $\delta^{15} \mathrm{~N}$ da parte aérea de leguminosas, dependendo de fixação do $\mathrm{N}_{2}$ para o crescimento diminui com o $\mathrm{N}$ acumulado, mostrando um padrão semelhante ao da curva de diluição $\% \mathrm{~N}$ durante o desenvolvimento (Figura 5). Isto poderia sugerir que a diferença de $\delta^{15} \mathrm{~N}$ com o crescimento da planta também pode ser explicada como uma função do taxa de tecido (por exemplo, relação folha/colmo). 


$$
\begin{aligned}
& \text { atom } \%{ }^{15} N=\left(\frac{{ }^{15} N}{{ }^{14} N+{ }^{15} N}\right) * 100 \\
& \delta^{15} N(\%)=\left(\frac{\text { atom } \%{ }^{15} N-0.3663}{0,3663}\right) * 1000
\end{aligned}
$$

Figura 4. As unidades de abundância isotópica. A abundância de isótopos estáveis de $\mathrm{N}$ são expressos em termos de $\%$ de átomos ${ }^{15} \mathrm{~N}$ ou $\delta^{15} \mathrm{~N}$. Átomo $\%{ }^{15} \mathrm{~N}$ é a percentagem do isótopo ${ }^{15} \mathrm{~N}$ menor em relação ao total de N. A unidade $\delta{ }^{15} \mathrm{~N}$ é frequentemente utilizada para ilustrar a diferença em abundância ${ }^{15} \mathrm{~N}$ entre um composto e um padrão em partes por mil. A composição atmosférica $\mathrm{N}_{2}\left(\mathrm{AIR}-\mathrm{N}_{2}\right)$ é o maior pool de $\mathrm{N}$ no meio ambiente (4\% do nitrogênio total terra) e é o padrão de referência internacional para medições de isótopos de nitrogênio. Assim, os valores de $\delta{ }^{15} \mathrm{~N}$ descrevem o desvio de uma amostra em relação à composição isotópica de $\mathrm{N}^{2}$ atmosférico, com um átomo de $15 \mathrm{~N} \%$ de $0,3663 \%$. Padrões isotópicos é atribuído o valor 0; $\delta{ }^{15} \mathrm{~N}$ de $\mathrm{N}_{2}$ atmosférico é de $0 \%$.

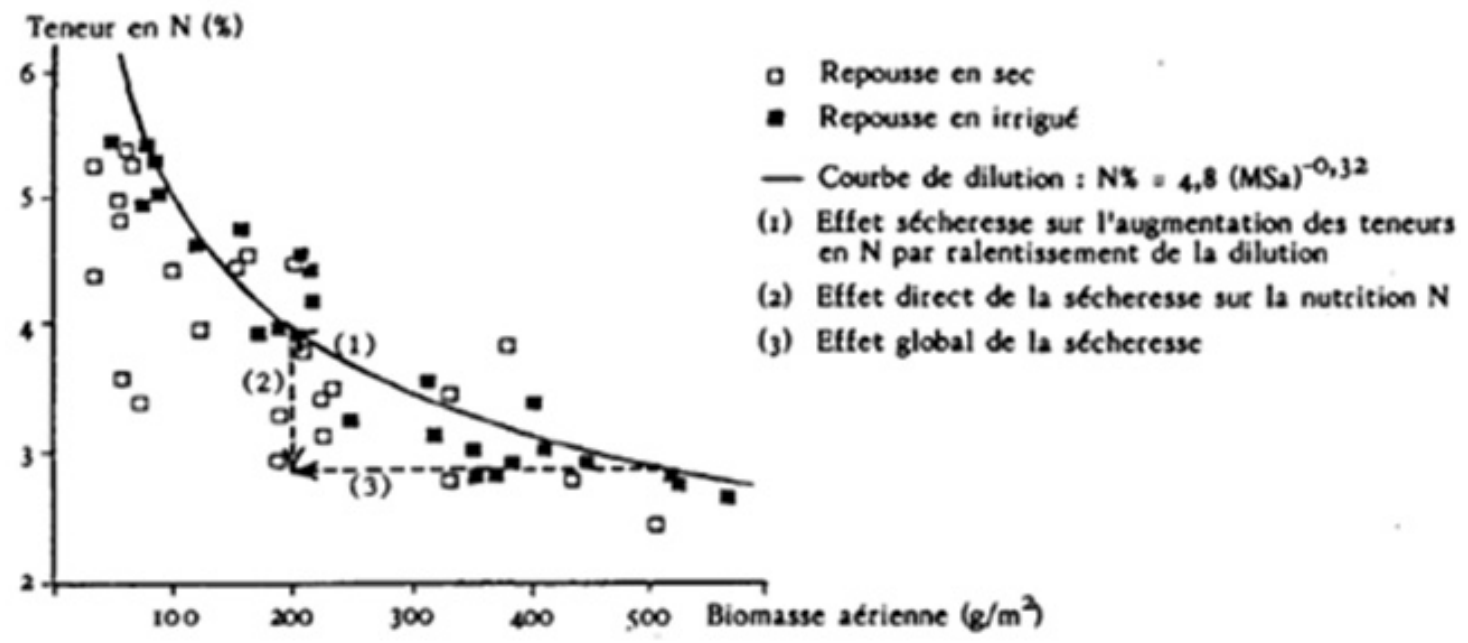

Figura 5. Mudanças no conteúdo de $\mathrm{N}$ de alfafa em função do acúmulo de matéria seca acima do solo durante a rebrota em condições irrigadas ou de sequeiro. (LEMAIRE e ALLIRAND, 1993).

\section{Conclusão}

Há vantagens e desvantagens associadas aos diferentes procedimentos usados. Os métodos desenvolvidos para determinar a fixação de $\mathrm{N}_{2}$ por associação de bactérias não foram exaustivamente testados em condições de campo. E estudos da distribuição da abundância natural de ${ }^{15} \mathrm{~N}$ do perfil do solo são também necessários. Assim, o estudo do fracionamento isotópico de $\mathrm{N}$ serve para o aprofundamento dos estudos sobre a dinâmica de transferência de $\mathrm{N}$ entre espécies de plantas e testar se as diferenças resultaram de diferença na fixação, proporção legume, dinâmica populacional ou composição do tecido, entre outros.

\section{Referências}

BERGERSEN, F.; G. TURNER. An evaluation of ${ }^{15} \mathrm{~N}$ methods for estimation nitrogen fixation in a subterranean clover-perennial ryegrass sward. Australian Journal of Agricultural Research. v.34, n.4, p. 391-401, 1983.

BODDEY, R.M., PEOPLES, M.B., PALMER, B.; DART, P.J. Use of the ${ }^{15} \mathrm{~N}$ natural abundance technique to quantify biological nitrogen fixation by woody perennials. Nutrient Cycling in Agroecosystems. v.57, p.235270,2000 . 


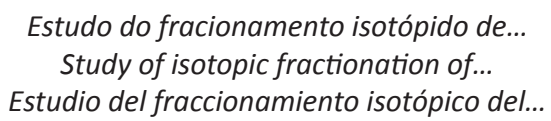

BODDEY, R. M.; URQUIAGA, S. Elephant grass genotypes for bioenergy production by direct biomass combustion. Pesquisa Agropecuária Brasileira, Brasília, v.44, n.2, p.133-140, fev. 2009.

BODDEY, R. M. Contribution of biological nitrogen fixation to Elephant grass (Pennisetum purpureum Schum.). Plant and soil, DOI 10.1007/s11104-011-0944-2, 2011.

CERETTA, J.C.; GIROTTO, E. Contexto para definir manejo de nitrogênio no sistema plantio direto. In: Fertilidade do solo e nutrição de plantas no sistema plantio direto. ASSOCIAÇÃO DOS ENGENHEIROS AGRÔNOMOS DOS CAMPOS GEREAIS. Universidade Estadual de Ponta Grossa, PR, 2011.

CHIKARAISHI, Y., MATSUMOTO, K., OGAWA, N.O., SUGA, H., KITAZATO, H., OHKOUCHI, N. Hydrogen, carbon and nitrogen isotopic fractionations durin chlorophyll biosynthesis in $\mathrm{C}_{3}$ higher plants. Phytochemistry. v.66, p. 911-920, 2005.

DOBEREINER, J. Nitrogen-fixing bacteria of the genus Beijerinchia Derx in the rhizosphere of sugarcane. Plant and Soil, v.15, p.211-217, 1961.

EPSTEIN, E.; BLOOM, A.J. Mineral nutrition of plants: principles and perspectives. 2. ed. Sunderland: Sinouer Associates, 2005.

EVANS, R.D. Physiological mechanisms influencing plant nitrogen isotope composition. Trends Plant Sci., v.6, n.3, p.121-126, 2001.

FRY, B. Stable Isotope Ecology. Springer, Berlin. 2006.

HARE, P.E., FOGEL, M.L., STAFFORD JR., T.W., MITCHELL, A.D. AND HOERING, T.C. The isotopic composition for carbon and nitrogen in individual amino acids isolated from modern and fossil proteins. Journal of Archaeological Science. v. 18, p.277-292, 1991.

KYEI-BOAHEN, S., SLINKARD, A.E.; WALLEY, F.L. Isotopic fractionation during $\mathrm{N}_{2}$ fixation by chickpea. Soil Biology \& Biochemistry. v.34, p. 417-420, 2002.

LEA, P.J. AND MOROT-GAUDRY, J.F. Nitrogen, Plant Growth and Crop Yield, Nodule Formation and Function. In Plant Nitrogen. Springer-Verlag Berlin, INRA Paris. p.101-146 and 343-367, 2001.

LEMAIRE, G. AND ALLIRAND, J.M. Relation entre croissance et qualité de la luzerne : interaction génotypemode d'exploitation. Fourrages. v.134, p.183-198, 1993.

LEMAIRE, G. AND GASTAL, F. N Uptake and Distribution in Plant Canopies. In Diagnosis of the Nitrogen Status in Crops. Springer-Verlag Berlin. p. 3-43, 1997.

LOUARN, G., CORRE-HELLOU, G., FUSTEC, J., LO-PELZER, E., JULIER, B., LITRICO, I., HINSINGER, P., LECOMTE, C. Déterminants écologiques et physiologiques de la productivité et de la stabilité des associations graminées-légumineuses. Innovations Agronomiques. v.11, p.79-99, 2010.

MARIOTTI, F., CHAMPIGNY, M.L., AMARGER, N. AND MOYSE, A. Nitrogen isotope fractionation associated with nitrate reductase activity and uptake of $\mathrm{N}$ by pearl millet. Plant Physiol. v.69, p.880-884, 1982.

MAURIES, M. Luzerne : culture, récolte, conservation, utilisation. Editions France Agricole. 2003.

MORAIS, R. F.; SOUZA, R. J.; LEITE, J. M.; SOARES, L. H. B.; ALVES, B. J. R.;

MORAIS, R. F.; QUESADA, D. M.; REIS, V. M.; URQUIAGA, S.; ALVES, B. J. R.;

SHEARER, G., AND KOHL, D. H. N2-Fixation in Field Settings: Estimations Based on Natural ${ }^{15} \mathrm{~N}$ Abundance. Aust. J. Plant Physiol. v.13, p.699-756, 1986.

STEELE, K.W., BONISH, P.M., DANIEL, R.M. AND O'HARA, G.W. Effect of Rhizobial Strain and Host Plant on Nitrogen Isotopic Fractionation in Legumes. Plant Physiol.v.72, p.1001-1004, 1983.

SUTTON, M.A. AND VAN GRINSVEN, H. The European nitrogen problem in a global perspective, Nitrogen processes in terrestrial ecosystems. In The European Nitrogen Assessment. Cambridge: Cambridge University Press. p.9-31 and 99-12, 2011. 
TJEPKEMA, J.D., SCHWINTZER, C.R., BURRIS, R.H., JOHNSON, G.V. AND SILVESTER, W.B. Natural abundance of 15N in actinorhizal plants and nodules. Plant and Soil.v.219, p.285-289, 2000.

UNKOVICH, M. J., PATE, J. S., SANFORD, P. AND ARMSTRONG, E. L. Potential precision of the d15N natural abundance method in field estimates of nitrogen fixation by crop and pasture legumes in S.W. Australia. Austral. J. of Agri. Res. v.45, p.119-132, 1994.

UNKOVICH, M., HERRIDGE, D., PEOPLES, M., CADISCH, G., BODDEY, R., GILLER, K., ALVES, B. AND CHALK, P. Measuring plant-associated nitrogen fixation in agricultural systems. Clarus design, Canberra. 2008.

URQUIAGA, S.; XAVIER, R. P.; MORAIS, R. F.; BATISTA, R. B.; SCHULTZ, N.; LEITE, J. M.; SÁ, J. M.; BARBOSA, K.P.; RESENDE, A. S.; ALVES, B. J. R.; ODDEY, R. M. Evidence from field nitrogen balance and ${ }^{15} \mathrm{~N}$ natural abundance data for the contribution of biological $\mathrm{N}_{2}$ fixation to Brazilian sugarcane varieties. Plant Soil. p.3-17, 2011. DOI 10.1007/s11104-011-1016-3.

VITOUSEK, P.M.; FARRINGTON, H. Nutrient limitation and soil development: experimental testo f a biogeochemical theory. Biogeochemistry, Dortrecht, v. 37. 1997.

WATANABE, I.; YONEYAMA, T.; PADRE, B.; LADHA, J.K. Difference in natural abundance of ${ }^{15} \mathrm{~N}$ in several rice (Oriza sativa L.) varieties: Aplication for evaluating $\mathrm{N}_{2}$ fixation. Soil Science Plant Nutrition, $\mathrm{v}$. 33, p.407-415., 1987.

WERNER, R.A. AND SCHMIDT, H.L. The in vivo nitrogen isotope discrimination among organic plant compounds. Phytochemistry.v.61, p.465-484, 2002. 